Conclusions High plasma copeptin levels indicate a worse outcome in NSTEMI patients. We have demonstrated that copeptin fulfils AHA criteria by improving risk stratification over established markers GRACE score and NT-proBNP. Copeptin is also useful for rapid rule-out of $\mathrm{MI}$ and the current findings further support clinical uptake.

\section{THE RELATIONSHIP BETWEEN PSYCHOLOGICAL FACTORS AND IMPAIRED HEALTH-RELATED QUALITY OF LIFE POST ST-ELEVATION MYOCARDIAL INFARCTION}

doi:10.1136/heartjnl-2011-300198.12

${ }^{1} \mathrm{~L}$ McGowan, ${ }^{2} \mathrm{H}$ lles-Smith, ${ }^{1} \mathrm{C}$ Dickens, ${ }^{1} \mathrm{M}$ Campbell, ${ }^{1} \mathrm{C}$ Rogers, ${ }^{2} \mathrm{~F}$ Fath-Ordoubadi. ${ }^{1}$ University of Manchester, Manchester, UKI; ${ }^{2} \mathrm{CMFT}$, Manchester, UK

Introduction Evidence suggests that psychological factors, such as depression and anxiety, are independent risk factors for increased morbidity and mortality post ST-elevation myocardial infarction (STEMI). Since improved treatments have increased survival rates post STEMI the emphasis has turned to more patient related outcome measures such as health-related quality of life (HRQoL). The aim of the study was to assess the contribution of anxiety and depression to HRQoL in post STEMI patients, after controlling for possible confounding factors, including type of treatment.

Methods We conducted a prospective cohort study of 385 postSTEMI patients who had undergone either lysis (183) or PPCI (202). The mean age was 60.0 years (SD 11.8) and $78 \%$ were male. Patients were assessed on a range of demographic, clinical and psychosocial variables, including measures of cardiac risk, cardiac severity and comorbidity (Charlson Comorbidity IndexCCI). Psychosocial assessment included anxiety and depression (Hospital Anxiety and Depression Scale), illness perceptions (brief IPQ), and health-related quality of life (SF-36). The main outcome was the SF-36 Physical Component Score (PCS) at 6 months postSTEMI

Results Baseline results revealed a small number significant differences between groups on a range of clinical variables, including higher GRACE scores for PPCI group $(p=0.007)$ but no differences in LV function. Lysis patients had more comorbid illness as measured by the CCI ( $p=0.037)$. Regarding psychological variables the total HADS score was significantly higher in the PPCI vs lysis group at baseline (means 13.2 (SD 7.9) and 11.4 (SD 8.9), $\mathrm{p}=0.035$ ), while anxiety and depression almost reached significance, with raised anxiety and depression scores in the PPCI group. In order to identify variables at baseline that may contribute to SF-36 PCS at 6 months, we conducted a hierarchical multiple regression with four blocks of independent variables-demographic, comorbidity-related, clinical and psychological. Factors which contributed to the final model were cholesterol levels $(p=0.031)$ and depression $(p<0.001)$. Treatment group did not play a role $(\mathrm{p}=0.199)$. The addition of anxiety and depression contributed significantly to the reporting of lower physical health-related quality of life (PCS) at 6 months ( $\ddot{\mathrm{A}} 2=0.12$, $\mathrm{p}<0.001$ ).

Conclusion The findings have shown that raised levels of depression and anxiety predicted impairment in health-related quality of life at 6 months post-STEMI, regardless of mode of treatment. The results indicate that the assessment of psychological factors is important in both groups. Despite PPCI having improved clinical outcomes, there will always be a group of patients receiving lysis. As such it is important to assess anxiety and depression in post STEMI patients, and to include these potentially modifiable factors in the design of suitable interventions for this patient group.

\section{NEUTROPHIL ACTIVATION AT THE CULPRIT LESION IN ACUTE ST-SEGMENT ELEVATION MYOCARDIAL INFARCTION WITH MULTIPLE COMPLEX CORONARY PLAQUES}

doi:10.1136/heartjnl-2011-300198.13

${ }^{1} \mathrm{C} \mathrm{J}$ Marshall, ${ }^{1} \mathrm{~J}$ L Mckenzie, ${ }^{2} \mathrm{~T}$ Moccata, ${ }^{3} \mathrm{M}$ Nallaratnam, ${ }^{3} \mathrm{~J}$ Blake, ${ }^{3} \mathrm{C}$ Frampton, ${ }^{3} \mathrm{M}$ Richards, ${ }^{2} \mathrm{~A}$ J Kettle, ${ }^{3} \mathrm{D}$ R Mcclean. ${ }^{1}$ Sunderland Royal Hospital, Sunderland, UK; ${ }^{2}$ Free Radical Research Group, University of Otago, Christchurch, New Zealand; ${ }^{3}$ Department of Cardiology, Christchurch Hospital, Christchurch, New Zealand

Introduction The activation of neutrophils at the culprit coronary lesion following acute plaque disruption has not been reported. We hypothesised that neutrophil activation occurs in ST elevation myocardial infarction (STEMI) prior to percutaneous intervention (PCI), and that differences in activation may be detectable locally at the culprit lesion, particularly in patients with multiple complex coronary plaques.

Methods Forty STEMI patients having primary PCI were compared to 10 controls with chronic stable angina (CSA) undergoing elective PCI. The clinical, demographic and angiographic characteristics of patients and controls are shown in Abstract 13 table 1 . The culprit lesion was sampled after passage of a guide wire across the lesion and use of a low profile sampling catheter (Multifunctional probing catheter, Boston Scientific Corporation, Natick, Massachusetts, USA) at the site of occlusion, prior to further mechanical intervention. Neutrophil activation was measured by flow cytometry using neutrophil intracellular myeloperoxidase content (MPO Index) and the expression of the $\beta 2$ - integrin CD11b, a leukocyte adhesion and activation marker at the culprit coronary lesion (CA), the aorta at the coronary ostium (Ao), the coronary sinus (CS), and femoral artery (FA) prior to primary PCI. A lower $\mathrm{MPO}$ content indicates the depletion of intracellular MPO and cell activation.

\section{Abstract 13 Table 1}

\begin{tabular}{lllc}
\hline Variable & $\begin{array}{l}\text { STEMI } \\
(\mathbf{n = 4 0 )}\end{array}$ & $\begin{array}{l}\text { Elective PCI } \\
(\mathbf{n = 1 0})\end{array}$ & $\mathbf{p}$ Value \\
\hline Age (years) mean \pm SD & $62 \pm 12$ & $68 \pm 9.1$ & 0.9 \\
Male (\%) & $28(70)$ & $7(70)$ & 1 \\
$\begin{array}{l}\text { Culprit coronary artery lesion treated (\%) } \\
\text { Left anterior descending }\end{array}$ & $17(42)$ & $5(50)$ & 0.73 \\
Diagonal & $2(5)$ & $1(10)$ & 0.5 \\
Circumflex & $1(3)$ & $2(20)$ & 0.1 \\
Right coronary & $20(50)$ & $2(20)$ & 0.15 \\
Time to presentation (mins) mean $\pm S D$ & $222 \pm 155$ & NA & \\
Baseline TIMI flow 0-1 & $28(70)$ & 0 & $<0.001$ \\
\hline
\end{tabular}

Results A marked decrease in MPO content occurred at the CA, Ao and FA in STEMI compared to elective controls $(p<0.01)$. Furthermore, MPO content was lower at the CA $(-23.1,(-25.6$ to -17.1$)$, $\mathrm{n}=37)$ compared to Ao $(-22.0,(-24.7$ to -16.2$), \mathrm{n}=37)$, CS $(-20.6$, $(-24.8$ to -16.9$), \mathrm{n}=30)$ and FA $(-20.4,(-24.4$ to -13.1$), \mathrm{n}=40)$, all $\mathrm{p}<0.001$ (Abstract 13 figure 1). Neutrophil MPO content was correlated with CD11b expression only at the culprit CA in STEMI $(\mathrm{r}=-0.4, \mathrm{p}=0.03, \mathrm{n}=31$ ) (Abstract 13 figure 2). Neutrophil MPO content at the CA in patients with multiple complex plaques was similar to those with a single culprit however only in those with multiple complex plaques was a correlation between MPO content and CD11b ( $\mathrm{r}=-0.7, \mathrm{p}=0.02)$ shown. Conclusion: In acute STEMI, neutrophils are activated systemically, regionally and locally at the culprit coronary lesion. In patients with multiple complex plaques, there may be an extended local role for the activated neutrophil following acute plaque destabilisation. 